\title{
ON THE CONVOLUTION EQUATIONS IN THE SPACE OF DISTRIBUTIONS OF $L^{p}$-GROWTH
}

\author{
D. H. PAHK ${ }^{1}$
}

\begin{abstract}
We consider convolution equations in the space $D_{L^{p}}^{\prime}, 1 \leqslant p \leqslant \infty$, of distributions of $L^{p}$-growth, i.e. distributions which are finite sums of derivatives of $L^{p}$-functions (see $\left.[4,7]\right)$. Our main results are to find a condition for convolution operators to be hypoelliptic in $\mathscr{D}_{L^{\infty}}^{\prime}$ in terms of their Fourier transforms and to show that the same condition is working for the solvability of convolution operators in the tempered distribution space $\mathscr{S}^{\prime}$ and $\mathscr{D}_{L^{p}}^{\prime}$.
\end{abstract}

Preliminary. We recall the basic facts about the spaces $\mathscr{D}_{L^{p}}^{\prime}, 1 \leqslant p \leqslant \infty$, and $\mathscr{S}^{\prime}$, which we need in this paper. For the proof we refer to $[4,7]$.

The space $\mathscr{D}_{L^{p}}^{\prime}, 1 \leqslant p \leqslant \infty$. Let $\mathscr{D}_{L^{p}}^{\prime}$ be the space of all $C^{\infty}$-functions $\phi$ in $\mathbf{R}^{n}$ such that $\mathscr{D}^{\alpha} \phi$, for all $\alpha \in \mathbf{N}^{n}$, is in $L^{p}\left(\mathbf{R}^{n}\right)$ equipped with the topology generated by countable norms

$$
\|\phi\|_{m, p}=\left\{\sum_{|\alpha| \leqslant m}\left\|D^{\alpha} \phi\right\|_{L^{p}}^{p}\right\}^{1 / 2}, \quad m \in \mathbf{N}, 1 \leqslant p<\infty,
$$

and

$$
\|\phi\|_{m, \infty}=\sup _{|\alpha| \leqslant m}\left\|D^{\alpha} \phi\right\|_{L^{\infty}}, \quad m \in \mathbf{N} .
$$

Then it is obviously a Fréchet space and a normal space of distributions in $\mathbf{R}^{n}$. We also have $C_{c}^{\infty} \subset \mathscr{D}_{L^{p}} \subset \mathscr{D}^{\prime}$ with continuous injections.

We denote by $\mathscr{D}_{L^{p}}^{\prime}, 1 \leqslant p \leqslant \infty$, the dual of $\mathscr{D}_{L^{q}}$, where $1 / p+1 / q=1$ and these duals are subspaces of the space of distributions in $\mathbf{R}^{n}$. A distribution $T$ is in $\mathscr{D}_{L^{p}}^{\prime}$, $1 \leqslant p \leqslant \infty$, if and only if there is an integer $m(T)>0$ such that

$$
T=\sum_{|\alpha| \leqslant m} D^{\alpha} f_{\alpha}, \quad \alpha \in \mathbf{N}^{n},
$$

where the $f_{\alpha}$ 's are bounded continuous functions belonging to $L^{p}\left(\mathbf{R}^{n}\right)$. Moreover, if $p<\infty$, each $f_{\alpha}$ converges to zero at infinity.

The Fourier transform of a function in $\mathscr{D}_{L^{1}}$ is a continuous function rapidly decreasing at infinity and also the Fourier transform of a distribution in $\mathscr{D}_{L^{1}}^{\prime}$ is a continuous function slowly increasing at infinity.

Received by the editors November 28, 1983 and, in revised form, April 24, 1984.

1980 Mathematics Subject Classification. Primary 35D10, $35 \mathrm{H} 05$.

${ }^{1}$ Partly supported by Korean Science and Engineering Foundation. 
The space $\mathscr{S}^{\prime}$. Let $\mathscr{S}$ be the space of all $C^{\infty}$-functions $\phi$ in $\mathbf{R}^{n}$ such that

$$
\sup _{|\alpha| \leqslant k, x \in \mathbf{R}^{n}}(1+|x|)^{k}\left|D^{\alpha} \phi(x)\right|<\infty, \quad k=0,1,2, \ldots,
$$

equipped with the topology generated by these countable norms. We denote by $\mathscr{S}^{\prime}$ the dual of $\mathscr{S}$. The Fourier transformation is now an isomorphism of $\mathscr{S}$ onto itself and of $\mathscr{S}^{\prime}$ onto $\mathscr{S}^{\prime}$.

The space $\mathcal{O}_{c}^{\prime}\left(\mathscr{S}^{\prime}: \mathscr{S}^{\prime}\right)$ of convolution operators in $\mathscr{S}^{\prime}$ consists of distributions $S \in \mathscr{S}^{\prime}$ satisfying one of the following equivalent conditions:

(i) Given any $k=1,2, \ldots, S$ can be represented in the form

$$
S=\sum_{|\alpha| \leqslant m} D^{\alpha} f_{\alpha}
$$

where $f_{\alpha},|\alpha| \leqslant m$, are continuous functions in $\mathbf{R}^{n}$ such that

$$
f_{\alpha}(x)=O\left((1+|x|)^{-k}\right) \quad \text { as }|x| \rightarrow \infty .
$$

(ii) For every $\phi$ in $\mathscr{S}, S * \phi$ is in $\mathscr{S}$. Moreover, the mapping $\phi \rightarrow S * \phi$ of $\mathscr{S}$ into $\mathscr{S}$ is continuous.

The Fourier transform $\hat{S}$ of a distribution $S$ in $\mathcal{O}_{c}^{\prime}\left(\mathscr{S}^{\prime}: \mathscr{S}^{\prime}\right)$ is a $C^{\infty}$-function with the following property: For every multi-index $\alpha$ there exists a nonnegative integer $l$ such that

$$
D^{\alpha} \hat{S}(\xi)=O\left((1+|\xi|)^{l}\right) \quad \text { as }|\xi| \rightarrow \infty .
$$

We denote by $\mathcal{O}_{M}\left(\mathscr{S}^{\prime}: \mathscr{S}^{\prime}\right)$ the space of all $C^{\infty}$-functions with the above property (3). They are multiplication operators in $\mathscr{S}^{\prime}$ and the Fourier transformation is an isomorphism of $\mathscr{O}_{c}^{\prime}\left(\mathscr{S}^{\prime}: \mathscr{S}^{\prime}\right)$ onto $\mathcal{O}_{M}\left(\mathscr{S}^{\prime}: \mathscr{S}^{\prime}\right)$ (see [7, Volume II]).

Hypoelliptic convolution equations in the space $\mathscr{D}_{L^{p}}^{\prime}, 1 \leqslant p \leqslant \infty$. In [10], Zielézny showed how to define, in a general manner, hypoelliptic and entire elliptic convolution operators in subspace of the space of distributions. He also characterized hypoelliptic and entire elliptic convolution operators in the space $\mathscr{S}^{\prime}$ of tempered distributions. In [6 and 12], he studied hypoelliptic convolution operators in the space of distributions of exponential growth of polynomial power and, in [5], Pahk studied the same problem in the space of distributions of generalized exponential growth introduced in [2].

In this paper it can be seen that for a distribution $S$ in $\mathcal{O}_{c}^{\prime}$ the hypoellipticity of the convolution operator $S$ in the space of tempered distributions is equivalent to the hypoellipticity in the space of bounded distributions. We define hypoelliptic convolution operators in $\mathscr{D}_{L^{\infty}}^{\prime}$ as follows: A distribution $S$ in $\mathscr{D}_{L^{1}}^{\prime}$ is said to be hypoelliptic in $\mathscr{D}_{L^{\infty}}^{\prime}$, if every solution $U$ in $\mathscr{D}_{L^{\infty}}^{\prime}$ of the convolution equation

$$
S * U=V
$$

is in $\mathscr{D}_{L^{\infty}}$, when $V$ is in $\mathscr{D}_{L^{\infty}}$; in that case equation (1) is also called hypoelliptic in $\mathscr{D}_{L^{x}}^{\prime}$. Since the space of convolution operators in $\mathscr{D}_{L^{\infty}}^{\prime}$ is $\mathscr{D}_{L^{1}}^{\prime}$, hypoelliptic convolution operators in $\mathscr{D}_{L^{\infty}}^{\prime}$ has to be characterized in $\mathscr{D}_{L^{1}}^{\prime}$. Because of lack of differentiability of their Fourier transforms there are some difficulties to achieve our goal. In 
this paper we only consider subclasses of $\mathscr{D}_{L^{1}}^{\prime}$, containing $\mathcal{O}_{c}^{\prime}$, whose Fourier transforms have certain order derivatives and increase slowly at infinity. In this class we can characterize hypoelliptic convolution operators in $\mathscr{D}_{L^{\infty}}^{\prime}$. But we have an example of hypoelliptic convolution operators in $\mathscr{D}_{L^{\infty}}^{\prime}$ which is not in this class.

We now establish a necessary and sufficient condition for a convolution operator to be hypoelliptic in $\mathscr{D}_{L^{\infty}}^{\prime}$. The result is proved only for a subclass of convolution operators in $\mathscr{D}_{L^{\infty}}^{\prime}$ and the proof is based on an idea similar to that used in [10 and 12].

Definition. $S \in \mathscr{D}_{L^{1}}^{\prime}$ is said to be of class $H_{m}$ if the Fourier transform $\hat{S}$ is a $C^{m}$-function in $\mathbf{R}^{n}$ and $D^{\alpha} S,|\alpha| \leqslant m$, are slowly increasing at infinity.

The fact that the Fourier transform is a topological isomorphism from $\mathcal{O}_{c}^{\prime}$ onto $\mathcal{O}_{M}$ (see [1, Chapter VII]) implies that every distribution in $\mathcal{O}_{c}^{\prime}$ is of class $H_{m}$. This class $H_{m}$ of distributions in $\mathscr{D}_{L^{1}}^{\prime}$ will be used for our study of hypoellipticity in $\mathscr{D}_{L^{\infty}}^{\prime}$. We begin with a lemma.

LEMMA. Let $S$ be a distribution whose Fourier transform is of the form

$$
\hat{S}=\sum_{j=1}^{\infty} a_{j} \delta\left(\xi_{j}\right),
$$

where the $\xi_{j}$ satisfy the condition

$$
\left|\xi_{j}\right|>2\left|\xi_{j-1}\right|>2^{j}, \quad j=1,2, \ldots,
$$

and the $a_{j}$ are complex numbers such that

$$
\left|a_{j}\right|=O\left(\left|\xi_{j}\right|^{\mu}\right) \quad \text { as } j \rightarrow \infty
$$

for some $\mu$; then the series in (5) converges in $\mathscr{D}_{L^{\infty}}^{\prime}$. We assert that $S \in \mathscr{D}_{L^{\infty}}$ if and only if

$$
\left|a_{j}\right|=O\left(\left|\xi_{j}\right|^{-\nu}\right) \text { as } j \rightarrow \infty
$$

for every $\nu>0$.

Proof. Using the fact that, for $\phi \in \mathscr{D}_{L^{1}}$

$$
\left|\xi^{\alpha} \hat{\phi}(\xi)\right| \leqslant\left\|D^{\alpha} \phi\right\|_{L^{1}}, \quad \alpha \in \mathbf{N}^{n},
$$

the Fourier transforms of functions in a bounded set in $\mathscr{D}_{L^{1}}$ are uniformly $O\left(|\xi|^{-\nu}\right)$ as $|\xi| \rightarrow \infty$, for every $\nu>0$. Therefore the series $S=\sum_{j=1}^{\infty} a_{j} e^{i\left\langle x, \xi_{j}\right\rangle}$ converges in $\mathscr{D}_{L^{\infty}}^{\prime}$. If the $a_{j}$ satisfy the condition (8), then the last series and all its term-by-term derivatives converge uniformly in $\mathbf{R}^{n}$. Consequently, $S$ is a $C^{\infty}$-function bounded together with its derivatives and so belongs to $\mathscr{D}_{L^{\infty}}$. The converse proof is exactly the same in [10].

We are now in a position to prove our main theorem.

THEOREM 1. Let $S$ be a distribution in $\mathscr{D}_{L^{1}}^{\prime}$ which is of class $H_{m}, m>n$. Then $S$ is hypoelliptic in $\mathscr{D}_{L^{\infty}}^{\prime}$ if and only if its Fourier transform satisfies the following condition: There are constants $a$ and $M$ such that

$$
|\hat{S}(\xi)| \geqslant|\xi|^{a} \text { for } \xi \in \mathbf{R}^{n} \text { and }|\xi| \geqslant M .
$$


Proof. Suppose that the condition (9) is not satisfied. Then there exists a sequence $\xi_{j}$ in $\mathbf{R}^{n}$ defined as in the Lemma and such that

$$
\left|\hat{S}\left(\xi_{j}\right)\right|<\left|\xi_{j}\right|^{-j}, \quad j=1,2, \ldots
$$

Then the series

$$
U=\sum_{j=1}^{\infty} e^{i\left\langle x, \xi_{j}\right\rangle}
$$

converges in $\mathscr{D}_{L^{\infty}}^{\prime}$, but by the Lemma $U$ is not in $\mathscr{D}_{L^{\infty}}$. On the other hand,

$$
S * U=\sum_{j=1}^{\infty} \hat{S}\left(\xi_{j}\right) e^{i\left\langle x, \xi_{j}\right\rangle},
$$

and applying the Lemma we conclude that $S * U$ is in $\mathscr{D}_{L^{\infty}}$. Thus $S$ is not hypoelliptic in $\mathscr{D}_{L^{\infty}}^{\prime}$.

Conversely, let us take a $C^{\infty}$-function $\psi$ in $\mathbf{R}^{n}$ such that

$$
\psi(\xi)= \begin{cases}1 & \text { for }|\xi|<M, \\ 0 & \text { for }|\xi|>M+1,\end{cases}
$$

where $M$ is the constant in (9). Then we define the Fourier transform $\hat{P}$ of $P$ by the formula

$$
\hat{P}(\xi)= \begin{cases}0 & \text { for }|\xi|<M, \\ \frac{1-\psi(\xi)}{\hat{S}(\xi)} & \text { for }|\xi| \geqslant M .\end{cases}
$$

Obviously $\hat{S}$ is a $C^{m}$-function slowly increasing together with its derivatives up to the $m$ th order. From the fact that $S$ is of class $H_{m}$ and (9) we can choose a positive integer $k$ so large that

$$
\hat{Q}(\xi)=\frac{1}{\left(1+|\xi|^{2}\right)^{k}} \hat{P}(\xi)
$$

and $D^{\alpha} \hat{Q}(\xi),|\alpha| \leqslant m$, are in $L^{1}\left(\mathbf{R}^{n}\right)$ and vanish at infinity, which follows from the iterated "chain rule"

$$
\partial^{\alpha}\left(\frac{1}{\hat{S}}\right)=\sum \frac{\pi_{1}^{k} \partial^{\alpha} \hat{S}}{\hat{S}^{k+1}} C_{\alpha_{1} \cdots \alpha_{k}} ; \quad \alpha_{1}+\cdots+\alpha_{k}=\alpha .
$$

Then we have, applying integration by parts,

$$
\begin{aligned}
|Q(x)| & =\left|\frac{1}{(2 \pi)^{n / 2}} \int_{\mathbf{R}^{n}} e^{i\langle x, \xi\rangle} \hat{Q}(\xi) d \xi\right| \\
& =\left|\frac{1}{(2 \pi)^{n / 2}} \frac{1}{\left(1+|x|^{2}\right)^{m / 2}} \int_{\mathbf{R}^{n}} e^{i\langle x, \xi\rangle}(1-\Delta)^{m / 2} \hat{Q}(\xi) d \xi\right| \\
& <C \frac{1}{\left(1+|x|^{2}\right)^{m / 2}} \quad \text { for some constant } C
\end{aligned}
$$


Therefore $Q(x)$ is an $L^{1}$-function, and so the distribution

$$
P=\left(1+D_{1}^{2}+\cdots+D_{n}^{2}\right)^{k} Q(x)
$$

is in $\mathscr{D}_{L^{1}}^{\prime}$. Furthermore $\hat{S}(\xi) \hat{P}(\xi)=1-\psi(\xi)$, whence, passing to the inverse Fourier transform, we see that $P$ is a rapidly decreasing parametrix for $S$ with $\hat{W}=\psi$, that is,

$$
S * P=\delta-W
$$

Now assume that $S * U=V$, where $V \in \mathscr{D}_{L^{\infty}}$ and $U \in \mathscr{D}_{L^{\infty}}^{\prime}$. Then, making use of (12), we can write

$$
\begin{aligned}
U & =U * \delta=U *(S * P)+U * W \\
& =(U * S) * P+U * W \\
& =V * P+U * W .
\end{aligned}
$$

It is obvious that $V * P$ and $U * W$ are in $\mathscr{D}_{L^{\infty}}$, so that $U$ is in $\mathscr{D}_{L^{\infty}}$.

Corollary. With the same hypothesis of $S$ in the theorem, (9) implies that every solution $U$ in $\mathscr{D}_{L^{p}}^{\prime}, 1 \leqslant p \leqslant \infty$, of the equation (4) is in $\mathscr{D}_{L^{p}}$ whenever $V$ is in $\mathscr{D}_{L^{p}}$.

Proof. Viewing the proof of sufficiency of the theorem, $P$ is in $\mathscr{D}_{L^{1}}^{\prime}$ and $U=V * P+U * W$. We can easily see the $\mathscr{D}_{L^{p}} * \mathscr{D}_{L^{1}}^{\prime} \subset \mathscr{D}_{L^{p}}$ and so $U$ is in $\mathscr{D}_{L^{p}}$.

If the given convolution operator $S$ is in $\mathscr{D}_{L^{1}}^{\prime}$, then we have the following weak version of the regularity theorem.

THEOREM 2. If a distribution $S$ in $D_{L^{1}}^{\prime}$ satisfies the condition (9), then every solution $U$ in $\mathscr{D}_{L^{\infty}}^{\prime}$ of the equation (1) with $V \in \mathscr{D}_{L^{2}}$ is in $\mathscr{D}_{L^{\infty}}$.

Proof. Applying the same argument as in Theorem 1, we construct the continuous function $\hat{P}(\xi)$ slowly increasing at infinity, and so we find a positive integer $k$ so large that

$$
\hat{Q}(\xi)=\frac{1}{\left(1+|\xi|^{2}\right)^{k}} \hat{p}(\xi)
$$

is in $L^{2}\left(\mathbf{R}^{n}\right)$. By Plancherel's theorem, $Q(x)$ is in $L^{2}\left(\mathbf{R}^{n}\right)$, and so the distribution $P=\left(1+D_{1}^{2}+\cdots+D_{n}^{2}\right)^{k} Q$ is in $\mathscr{D}_{L^{2}}^{\prime}$. Also, we have

$$
U=U * \delta=V * P+U * W
$$

Since $V$ is in $\mathscr{D}_{L^{2}}, V * P$ and $U * W$ are in $\mathscr{D}_{L^{\infty}}$, so that $U$ is in $\mathscr{D}_{L^{\infty}}$.

Combining Theorem 1 with the results of [10] we can state

THEOREM 3. Let $S$ be a distribution in $\mathcal{O}_{c}^{\prime}$. Then the following are equivalent:

(a) $S$ is hypoelliptic in $\mathscr{S}^{\prime}$.

(b) $S$ is hypoelliptic in $\mathscr{D}_{L^{\prime}}^{\prime}$.

(c) There exist constants $a$ and $M$ such that

$$
|\hat{S}(\xi)| \geqslant|\xi|^{a} \quad \text { for } \xi \in \mathbf{R}^{n} \text { and }|\xi| \geqslant M .
$$

We now give two examples of hypoelliptic convolution operators, one of which is not of class $H_{m}$. 
EXAMPLE 1. Let $S=e^{-|t|}$ in $R^{1}$. Since $\hat{S}(\xi)=1 /\left(1+\xi^{2}\right)$, it is in $\mathcal{O}_{c}^{\prime}$ and satisfies the condition (9). Therefore, it is hypoelliptic in $\mathscr{S}^{\prime}$ and $\mathscr{D}_{L^{x}}^{\prime}$.

EXAMPLE 2. Taking $S=1 /\left(1+x^{2}\right)+\delta$ in $R^{1}, \hat{S}(\xi)=e^{-|\xi|}+1$ is not a $C^{1}$. function in $\mathbf{R}^{1}$ and satisfies the condition (9) in Theorem 1 with $a=-1$ and $M=1$. From the fact that $1 /\left(1+x^{2}\right)$ is in $\mathscr{D}_{L^{1}}$ and $\mathscr{D}_{L^{1}} * \mathscr{D}_{L^{\infty}}^{\prime} \subset \mathscr{D}_{L^{\infty}}, S$ is a hypoelliptic convolution operator in $\mathscr{D}_{L^{\infty}}^{\prime}$.

REMARK. We can easily see that the convolution operator $S$ in $\mathcal{O}_{c}^{\prime}$ is solvable in $\mathcal{O}_{c}^{\prime}$ if and only if $\hat{S}$ satisfies the property (9) and has no zero in $\mathbf{R}^{n}$ and also characterizes the solvability in $\mathscr{D}_{L^{1}}^{\prime}$ because $S$ is actually invertible in $\mathcal{O}_{c}^{\prime}$. Therefore, the convolution operators in $\mathcal{O}_{c}^{\prime}$ which have the above properties are solvable in the spaces $\mathscr{D}_{L^{p}}^{\prime}$, $1 \leqslant p \leqslant \infty$, and $\mathscr{S}^{\prime}$, but the converse does not hold in general. We still leave the problems such as what condition guarantees the hypoellipticity in $\mathscr{D}_{L^{\infty}}^{\prime}$ for the general convolutors and the solvability in $\mathscr{D}_{L^{p}}^{\prime}, 1 \leqslant p \leqslant \infty$, and $\mathscr{S}^{\prime}$ without invertibility.

COMMENT. We appreciate the referee for various suggestions to reform our paper. He also suggested to study the relation between the number $m$ in the condition $\left(H_{m}\right)$ and the number $p$ which the Fourier transform of $\hat{S}(\xi)^{-1}\left(1+|\xi|^{2}\right)^{-k}$, for sufficiently large $k$, is an $L^{p}$-convolutor.

\section{REFERENCES}

1. L. Ehrenpreis, Solution of some problems of diversion. IV: Invertible elliptic operators, Amer. J. Math. 82 (1960), 522-588.

2. I. Gelfand and G. Shilov, Generalized functions, Vols. I, II, III, Academic Press, New York, 1968.

3. L. Hörmander, Hypoelliptic convolution equations, Math. Scand. 9 (1961), 178-184.

4. B. Neto, Introduction to the theory of distributions, Marcel Dekker, New York, 1973.

5. D. H. Pahk, Hypoelliptic convolution equations in $K_{M}^{\prime}$, Ph.D. Dissertation, State University of New York at Buffalo, 1981.

6. G. Sampson and Z. Zielézny, Hypoelliptic convolution equations in $\mathscr{K}_{p}^{\prime}, p>1$, Trans. Amer. Math. Soc. 223 (1976), 133-154.

7. L. Schwartz, Théorie des distributions, Hermann, Paris, 1959.

8. S. Sznajder and Z. Zielézny, On some properties of convolution operators in $K_{1}^{\prime}$ and $\mathscr{S}^{\prime}$, J. Math. Anal. Appl. 65 (1978), 543-554.

9. __ Solvability of convolution equations in $\mathscr{K}_{1}^{\prime}$, Proc. Amer. Math. Soc. 57 (1976), 103-106.

10. Z. Zielézny, Hypoelliptic and entire elliptic convolution equations in subspaces of the space of distributions. I, Studia Math. 28 (1967), 317-332.

11. On the space of convolution operators in $K_{1}^{\prime}$, Studia Math. 31 (1978), 219-232.

12. Hypoelliptic and entire elliptic convolution equations in subspaces of the space of distributions. II, Studia Math. 32 (1969), 47-59.

Department of Mathematics, Yonsei University, Seoul 120, Korea 